\title{
Characteristics of a Colistin-Resistant Escherichia coli ST695 Harboring the Chromosomally-Encoded mcr-1 Gene
}

\author{
Zhong Peng ${ }^{1,2,+} \oplus$, Zizhe $\mathrm{Hu}^{1,2,+}$, Zugang $\mathrm{Li}^{1,2}$, Xiaosong $\mathrm{Li}^{1,2}$, Chaoying Jia ${ }^{1,2}$, \\ Xiaoxue Zhang ${ }^{1,2}$, Bin Wu ${ }^{1,2} \mathbb{D}$, Huanchun Chen ${ }^{1,2}$ and Xiangru Wang ${ }^{1,2, * \mathbb{D}}$ \\ 1 State Key Laboratory of Agricultural Microbiology, College of Animal Science and Veterinary Medicine, \\ Huazhong Agricultural University, Wuhan 430070, China; pengzhong@mail.hzau.edu.cn (Z.P.); \\ huzizhede@webmail.hzau.edu.cn (Z.H.); lizugang@webmail.hzau.edu.cn (Z.L.); \\ lixiaosong@webmail.hzau.edu.cn (X.L.); jcy116178@webmail.hzau.edu.cn (C.J.); \\ zhangxuenian@webmail.hzau.edu.cn (X.Z.); wub@mail.hzau.edu.cn (B.W.); \\ chenhch@mail.hzau.edu.cn (H.C.) \\ 2 The Cooperative Innovation Center for Sustainable Pig Production, Huazhong Agricultural University, \\ Wuhan 430070, China \\ * Correspondence: wangxr228@mail.hzau.edu.cn; Tel.: +86-27-87287290 \\ + These authors contributed equally to this work.
}

Received: 23 October 2019; Accepted: 9 November 2019; Published: 12 November 2019

check for updates

\begin{abstract}
Enterobacteriaceae having chromosomally-encoded mcr-1 is rarely reported. In this study, we recovered a chromosomal mcr-1 carrying Escherichia coli, designated HeN100, from the feces of a diarrheal pig in China. Antimicrobial susceptibility testing showed that HeN100 was resistant to three aminoglycosides, twelve $\beta$-lactams including three carbapenems, one phenicol, two tetracyclines, two fluoroquinolones, nitrofurantoin, and colistin tested. Oxford Nanopore MinION sequencing revealed that the complete genomes of the multidrug resistant (MDR) HeN100 consisted of a single circular chromosome and five circular plasmids. Bioinformatical analysis determined HeN100 as ST695 and it contained many acquired resistance genes responsible for its MDR phenotypes, including colistin resistance $m c r-1$ and the carbapenem resistance $b l a_{\mathrm{NDM}-1}$, and most of these genes were located on plasmids. However, the mcr-1 was found on the chromosome, and it was located between an IS30-like element ISApl1 and a PAP2-like encoding gene. These three genes consisted of an "ISApl1-mcr-1-orf" segment and inserted in high AT-rich regions. Finally, we found the bla $a_{\text {NDM-1 }}$ was carried on an IncFII type conjugative plasmid. The conjugation frequency of this plasmid was $7.61 \pm 2.11 \times 10^{-5}$ per recipient, and its conjugation conferred resistance to carbapenems and other $\beta$-lactams, as well as aminoglycosides. The spread of this $m c r-1 / b l a_{\mathrm{NDM}-1}$-carrying E. coli ST695 represents a great concern of public health.
\end{abstract}

Keywords: Escherichia coli; ST695; antibiotic resistance; Oxford Nanopore MinION sequencing; chromosomally-encoded $m c r-1$; plasmid-carrying $b l a_{\mathrm{NDM}-1}$; plasmid conjugation

\section{Introduction}

Polymyxins are cationic polypeptides with molecules of $\sim 1200 \mathrm{Da}$ in mass that consist of a cyclic heptapeptide possessing a tripeptide side chain acylated at the $\mathrm{N}$ terminus by a fatty acid tail [1]. Recently, polymyxins are well-established antibiotics that have regained significant interest as a consequence of the increasing incidence of infections due to multidrug-resistant Gram-negative bacteria [2]. Through a mechanism of destabilizing the lipopolysaccharide and causing the disruption of bacterial membranes, polymyxins are active against many Gram-negative bacteria in both human 
and veterinary medicine, including Pseudomonas aeruginosa, Acinetobacter baumannii, Klebsiella spp., Escherichia coli, Salmonella spp., Klebsiella spp., and other Enterobacteriaceae [3]. Several members of polymyxins, including polymyxin B and colistin (polymyxin E), are recognized as the last resort antibiotics for treating infections caused by multidrug-resistant Gram-negative bacteria in clinical practice [4].

Colistin-resistant Enterobacteriaceae has become a great concern to global public health [5,6]. While several mechanisms of resistance have been elucidated, the most important one is mediated by the transferrable $\mathrm{mcr}-1$ gene, which encodes the phosphoethanolamine transferase and it could be rapidly disseminated through the conjugative plasmid [7]. The plasmid-mediated mor- 1 has been reported globally in different bacterial species from different hosts since the report of a such transferrable $m c r-1$ in China in late 2015 [8]. In most $m c r$-1-bearing bacteria, the transferrable $m c r-1$ is generally located in IncX4-, IncI2-, IncHI2-, IncP-, IncFII-, and IncY-type plasmids [9]. However, there are comparably few articles having reported the chromosomally-encoded $m c r-1$ and its genetic basis is relatively limited [10]. In this study, the high-quality genome sequence of a colistin-resistant Escherichia coli harboring the chromosomally-encoded $m c r-1$ gene was sequenced through the Nanopore technology and together with the available genome sequences of some other E. coli which also harbors the chromosomally-encoded $m c r-1$, comparative analysis was performed to gain the knowledge on the genetic basis of the chromosome-born mor-1 as well as the other genetic characteristics of the chromosome-born mor-1 carrying E. coli.

\section{Materials and Methods}

\subsection{Bacterial Strains and Antimicrobial Susceptibility Testing}

E. coli HeN100 was isolated from the feces of a diarrheal pig in Henan Province in China, using MacConkey agar containing $2 \mu \mathrm{g} / \mathrm{mL}$ of colistin in combination of PCR detecting the $16 \mathrm{~S}$ rRNA gene and seven house-keeping genes ( $a d k, f u m C, g y r A, i c d, m d h, p u r A, r e c A)$ of $E$. coli [11]. The resistant phenotypes of the strain were determined using the broth microdilution method recommended by the United Sates Clinical \& Laboratory Standards Institute (CLSI M100, 28 ${ }^{\text {th }}$ Edition). A total of 28 types of antibiotics (Sigma-Aldrich, MO, USA) including amikacin (AMK), gentamicin (GEN), tobramycin (TOB), imipenem (IPM), meropenem (MRP), ertapenem (ETP), colistin (CL), cefazolin (CFZ), cefuroxime (CFX), cefoxitin (FOX), ceftazidime (CAZ), ceftriaxone (CRO), cefepime (CPM), chloramphenicol (CHL), fosfomycin (FOS), nitrofurantoin (NIT), ciprofloxacin (CIP), levofloxacin (LVX), moxifloxacin (MXF), norfloxacin (NOR), minocycline (MIN), tetracycline (TET), aztreonam (AZM), tigecycline (TGC), amoxicillin/clavulanate (AMC), ampicillin/sulbactam (AMS), piperacillin/tazobactam (PTZ), and trimethoprim/sulfamethoxazole (SXT) were tested. Results were interpreted using the CLSI breakpoints (CLSI M100, $28^{\text {th }}$ Edition). A European Committee on Antimicrobial Susceptibility Testing (EUCAST) breakpoint was also used for the interpretation when the CLSI breakpoint is not available. Each antibiotic was tested with three duplicates. E. coli ATCC 25,922 was used as quality control.

\subsection{Genome Sequencing, Assembly, and Annotation}

Genomic DNA of E. coli HeN100 was extracted using a QIAGEN Genomic-tip 500/G (QIAGEN, Duesseldorf, Germany). The quality and quantity of the bacterial genomic DNA was evaluated via electrophoresis on a $0.75 \%$ agarose gel and evaluation using a NanoDrop2000 (Thermo Scientific, Waltham, MA, USA) and Qubit 4 Fluorometer (Thermo Scientific, Waltham, USA). We applied an Oxford Nanopore Technology's (ONT) MinION sequencing technology for the SMRT sequencing. In briefly, $20 \mathrm{~kb} 30 \mathrm{~kb}$ DNA libraries were generated using an SQK-LSK109 Ligation Sequencing Kit (ONT, Oxford, United Kingdom) and were sequenced on a PromethION platform (ONT, Oxford, United Kingdom) at Nextomics Biosciences Co., Ltd. (Wuhan, China). The strategy yielded a total of $2,688,522,451$ base pairs (bp) raw data and a total of 2,686,893,251 bp filtered data were finally obtained after removing reads with mean_qscore_template $<7$ and length $<1000 \mathrm{bp}$. The filtered reads were 
de novo assembled using the Canu package (version 1.7.11) [12] with default parameters and the assembled data were fixed by pilon (version 1.22) [13] with default parameters, using the data obtained from the Illumina sequencing performed on an Illumina Hiseq Xten platform (Illumina Inc., San Diego, CA, USA) as the reference. The finally resulting genomes including the single circular chromosome and the circular plasmids were annotated using the best-placed reference protein set (GeneMarkS+) in the NCBI Prokaryotic Genome Annotation Pipeline (version. 3.3) [14]. The complete genome and the plasmids sequences of E. coli HeN100 have been deposited in the NCBI under accession numbers CP044443 (Chromosomal genome sequence of HeN100), CP044438 (Plasmid sequence of pHeN100-01), CP044439 (Plasmid sequence of pHeN100-02), CP044440 (Plasmid sequence of pHeN100-03), CP044441 (Plasmid sequence of pHeN100-04), CP044442 (Plasmid sequence of pHeN100-05).

\subsection{Bioinformatical Analysis}

Sequence-based bacterial serotyping was performed using SerotypeFinder (version 2.0) [15]. Antibiotic resistance genes were identified using blastn against a database generated from the Resfams database [16]. Acquired antimicrobial resistance genes as well as chromosomal mutations associated with resistant phenotypes were predicted using ResFinder (version 3.2) [17]. Bacterial sequence type was determined using Multi-Locus Sequence Typing 2.0 [18]. Insertion sequence (IS) elements were identified using ISfinder [19]. Unless otherwise specified, all the analyses in this study were conducted based on the alignment by MAFFT (version 7.429) [20].

The genome sequences of ten E. coli which also contained a chromosomal-bone mcr-1, including SZM489-1 (GenBank accession no. NIFQ01000000), SZM531-1 (GenBank accession no. NIFR01000000), SZM537-1 (GenBank accession no. NIFS01000000), SZM584-1 (GenBank accession no. NIFT01000000), SZM457-1 (GenBank accession no. NIFU01000000), SZH29-1 (GenBank accession no. NIFV01000000), SZH3951 (GenBank accession no. NIFW01000000), SZM334-1 (GenBank accession no. NIFX01000001), EC590 (GenBank accession no. NZ_CP016182), S51 (GenBank accession no. NZ_CP015995), as well as the genome sequence of E. coli MG1655 (GenBank accession no. U00096) were downloaded from NCBI. Seven E. coli ST696 sequences (ESC_NA6237, ESC_RA0580, ESC_WA7256, ESC_WA8549, ESC_WA8550, ESC_WA8551, ESC_ZA6085) were downloaded from the Enterobase database at https://enterobase.warwick.ac.uk. Comparative genomic analysis was performed and visualized using BLAST Ring Image Generator (BRIG) package [21] and EasyFig package (version 2.2.2) [22]. The average nucleotide identity (ANI) was calculated by ANI calculator [23]. The nucleotide sequences around the insertion sites of the mor-1 segments were compared and analyzed using WebLogo [24]. The evolutionary tree was generated by MEGA X [25] using the Maximum Likelihood method and Tamura-Nei model [26].

\subsection{Plasmid Conjugation}

Plasmid conjugation experiment was performed between the donor strain HeN100 which was resistant to imipenem and receipt strain $\mathrm{C} 600$ which was resistant to rifampicin, using the protocol described previously [27]. In briefly, overnight cultures of the donor strain in LB broth containing $40 \mu \mathrm{g} / \mathrm{mL}$ of imipenem and receipt strain in Luria Bertani (LB) Broth (Sigma-Aldrich, MO, USA) containing $1000 \mu \mathrm{g} / \mathrm{mL}$ of rifampicin were mixed together, centrifuged, and the bacterial pellets were resuspended using LB broth. After that, approximately $80 \mu \mathrm{L}$ of the bacterial resuspension was spotted onto a $1-\mathrm{cm}^{2}$ filter membrane which was pre-plated on the LB agar. The plate was then incubated for mating at $37^{\circ} \mathrm{C}$ for $12-18 \mathrm{~h}$. Finally, bacteria were washed from filter membrane and spread onto LB agars containing $40 \mu \mathrm{g} / \mathrm{mL}$ of imipenem and $1000 \mu \mathrm{g} / \mathrm{mL}$ of rifampicin for selection of $b l a_{\mathrm{NDM}-1}$-positive transconjugants. The donor and receipt bacteria were also streaked on the selective plates as control. The resistant phenotypes of the transconjugants were determined using broth microdilution method as mentioned above. 


\section{Results}

\subsection{Antimicrobial Susceptibility Profile of E. coli HeN100}

The antimicrobial susceptibility profile of E. coli HeN100 was tested using the broth microdilution method recommended by CLSI (CLSI M100, 28th Edition). The results revealed HeN100 was resistant to most classes of antibiotics tested, including three aminoglycosides (AMK, GEN, TOB), twelve $\beta$-lactams (ETP, IPM, MRP, CFZ, CFX, FOX, CAZ, CRO, CPM, AMC, AMS, PTZ), one phenicol (CHL), two tetracyclines (TET, MIN), two fluoroquinolones (MXF, CIP), one nitrofurantoin (NIT), and one polymyxin (CL) (Table 1). It was sensitive to the other two fluoroquinolones (LVX, NOR), fosfomycin, trimethoprim/sulfamethoxazole, and one $\beta$-lactam (AZM) (Table 1). In particular, the colistin-resistant HeN100 exhibited resistance to the three carbapenems including imipenem, meropenem, and ertapenem, simultaneously (Table 1).

Table 1. Resistance profile of E. coli HeN100.

\begin{tabular}{|c|c|c|c|c|c|}
\hline \multirow{2}{*}{ Antibiotics } & \multicolumn{2}{|c|}{ MIC Values $(\mu \mathrm{g} / \mathrm{mL})$ and Interpretation } & \multicolumn{3}{|c|}{ Interpretation Criteria $(\mu \mathrm{g} / \mathrm{mL})$} \\
\hline & HeN100 & ATCC25922 & S & $\mathbf{I}$ & $\mathbf{R}$ \\
\hline $\mathrm{CL} \ddagger$ & $>4(\mathrm{R})$ & $\leq 1(S)$ & $\leq 2$ & - & $>2$ \\
\hline $\mathrm{AMK} \S$ & $>32(\mathrm{R})$ & $\leq 8(S)$ & $\leq 16$ & 32 & $\geq 64$ \\
\hline GEN § & $>8(\mathrm{R})$ & $\leq 2(S)$ & $\leq 4$ & 8 & $\geq 16$ \\
\hline TOB $\S$ & $>8(\mathrm{R})$ & $\leq 2(\mathrm{~S})$ & $\leq 4$ & 8 & $\geq 16$ \\
\hline $\operatorname{IPM} \S$ & $>8(\mathrm{R})$ & $\leq 0.25(\mathrm{~S})$ & $\leq 1$ & 2 & $\geq 4$ \\
\hline $\mathrm{MRP} \S$ & $>8(\mathrm{R})$ & $\leq 0.13(\mathrm{~S})$ & $\leq 1$ & 2 & $\geq 4$ \\
\hline $\mathrm{ETP} \S$ & $>2(\mathrm{R})$ & $\leq 0.25$ (S) & $\leq 0.5$ & 1 & $\geq 2$ \\
\hline $\mathrm{CFZ} \S$ & $>16(\mathrm{R})$ & $\leq 2(\mathrm{~S})$ & $\leq 16$ & - & $\geq 32$ \\
\hline CFX§ & $>16(\mathrm{R})$ & $\leq 4(S)$ & $\leq 8$ & 16 & $\geq 32$ \\
\hline FOX $\S$ & $>16(\mathrm{R})$ & $\leq 4(\mathrm{~S})$ & $\leq 8$ & 16 & $\geq 32$ \\
\hline $\mathrm{CAZ}^{\S}$ & $>32(\mathrm{R})$ & $\leq 1(\mathrm{~S})$ & $\leq 4$ & 8 & $\geq 16$ \\
\hline $\mathrm{CRO}^{\S}$ & $>32$ (R) & $\leq 1(\mathrm{~S})$ & $\leq 1$ & 2 & $\geq 4$ \\
\hline $\mathrm{CPM}^{\S}$ & $>16(\mathrm{R})$ & $\leq 1(\mathrm{~S})$ & $\leq 2$ & - & $\geq 16$ \\
\hline $\mathrm{AMC}^{\S}$ & $>32 / 16(\mathrm{R})$ & $\leq 8 / 4(\mathrm{~S})$ & $\leq 8 / 4$ & $16 / 8$ & $\geq 32 / 16$ \\
\hline AMS \& & $>16 / 8(\mathrm{R})$ & $\leq 4 / 2(S)$ & $\leq 8 / 4$ & $16 / 8$ & $\geq 32 / 16$ \\
\hline PTZ § & $>64 / 4(\mathrm{R})$ & $\leq 4 / 4(\mathrm{~S})$ & $\leq 16 / 4$ & $32 / 4-64 / 4$ & $\geq 128 / 4$ \\
\hline $\mathrm{CHL}^{\S}$ & $>16(\mathrm{R})$ & $\leq 4(\mathrm{~S})$ & $\leq 8$ & 16 & $\geq 32$ \\
\hline MXF $\ddagger$ & $>2(\mathrm{R})$ & $\leq 0.5(\mathrm{~S})$ & $\leq 0.25$ & - & $>0.25$ \\
\hline $\mathrm{CIP} \S$ & $2(\mathrm{R})$ & $\leq 0.5(\mathrm{~S})$ & $\leq 1$ & 2 & $\geq 4$ \\
\hline LVX§ & $2(S)$ & $\leq 1(\mathrm{~S})$ & $\leq 2$ & 4 & $\geq 8$ \\
\hline NOR $\S$ & $4(S)$ & $\leq 2(S)$ & $\leq 4$ & 8 & $\geq 16$ \\
\hline TET § & $>8(\mathrm{R})$ & $\leq 2(S)$ & $\leq 4$ & 8 & $\geq 16$ \\
\hline $\mathrm{MIN} \&$ & $16(\mathrm{R})$ & $\leq 1(\mathrm{~S})$ & $\leq 4$ & 8 & $\geq 16$ \\
\hline TGC $\ddagger$ & 2 & $\leq 1(\mathrm{~S})$ & $\leq 1$ & - & $>2$ \\
\hline NIT $\S$ & $>64(\mathrm{R})$ & $\leq 16(S)$ & $\leq 32$ & 64 & $\geq 128$ \\
\hline FOS $\S$ & $\leq 16(\mathrm{~S})$ & $\leq 16(\mathrm{~S})$ & $\leq 64$ & 128 & $\geq 256$ \\
\hline SXT & $\leq 1 / 19(\mathrm{~S})$ & $\leq 1 / 19(\mathrm{~S})$ & $\leq 2 / 38$ & - & $\geq 4 / 76$ \\
\hline $\mathrm{AZM} \S$ & $\leq 2(S)$ & $\leq 2(S)$ & $\leq 4$ & 8 & $\geq 16$ \\
\hline
\end{tabular}

$\S$ Interpretation criteria and breakpoints referred to CLSI M100 ed28-2018. $\ddagger^{\ddagger}$ Interpretation criteria and breakpoints referred to EUCAST Clinical breakpoints-bacteria (v 8.1). R: Resistant; S: susceptible; I: intermediately resistant. CL: colistin; AMK: amikacin; GEN: gentamicin; TOB: tobramycin; IPM: imipenem; MRP: meropenem; ETP: ertapenem; CFZ: cefazolin; CFX: cefuroxime; FOX: cefoxitin; CAZ: ceftazidime; CRO: ceftriaxone; CPM: cefepime; AMC: amoxicillin/clavulanate; AMS: ampicillin/sulbactam; PTZ: piperacillin/tazobactam; CHL: chloramphenicol; MXF: moxifloxacin; CIP: ciprofloxacin; LVX: levofloxacin; NOR: norfloxacin; TET: tetracycline; MIN: minocycline; TGC: tigecycline; NIT: nitrofurantoin; FOS: fosfomycin; SXT: trimethoprim/sulfamethoxazole; AZM: aztreonam. 


\subsection{Genetic Basis for the Resistant Profile of E. coli HeN100}

The ONT MinION sequencing revealed that the multidrug resistant (MDR) HeN100 consisted of a single circular chromosome $(4,614,550 \mathrm{bp})$ and five circular plasmids $(114,090 \mathrm{bp}, 99,733 \mathrm{bp}, 83,958$ $\mathrm{bp}, 5277 \mathrm{bp}$, and $4665 \mathrm{bp}$ ). By using the sequenced-based serotyping and MLST typing method, HeN100 was assigned to O-antigen type: H-type O99:H8 and sequence type (ST) 695, respectively (Figure 1). The MDR ST695 strain HeN100 contained a number of resistance genes conferring resistance to macrolides $(m d f(A), m p h(A))$, quinolones (oqxA, oqxB, qnrS1), aminoglycosides (aadA1, aadA2, $\left.\operatorname{aph}\left(3^{\prime}\right)-I a, \operatorname{aph}\left(3^{\prime}\right)-V I, r m t B\right)$, phenicols $(c m l A 1, f l o R)$, tetracyclines $(\operatorname{tet}(A), \operatorname{tet}(M))$, trimethoprim $(\operatorname{dfr} A 12)$, colistin ( $m c r-1)$, and $\beta$-lactams (bla $\left.a_{\mathrm{NDM}-1}, b l a_{\mathrm{TEM}-105}, b l a_{\mathrm{TEM}-1 \mathrm{~B}}\right)$ (Figure 2). These resistance genes were located on the chromosome $(m d f(A), m c r-1)$, the 114-kb IncR type plasmid $(m p h(A)$, oqxA, oqxB, qnrS1, aadA1, aadA2, aph (3')-Ia, cmlA1, floR, tet $(A), \operatorname{tet}(M), \operatorname{dfr} A 12$, and bla ${ }_{\mathrm{TEM}-1 \mathrm{~B}}$ ) (Figure $\left.3 \mathrm{~A}\right)$, and the 83.9-kb IncFII type plasmid ( $\left.a p h\left(3^{\prime}\right)-V I, r m t B, b l a_{\mathrm{NDM}-1}, b l a_{\mathrm{TEM}-105}\right)$ (Figure 3B), respectively. However, most of these resistance genes were not found in other ST695 genomes (Figure 2). There were no chromosomal point mutations associated with resistance being identified in HeN100.

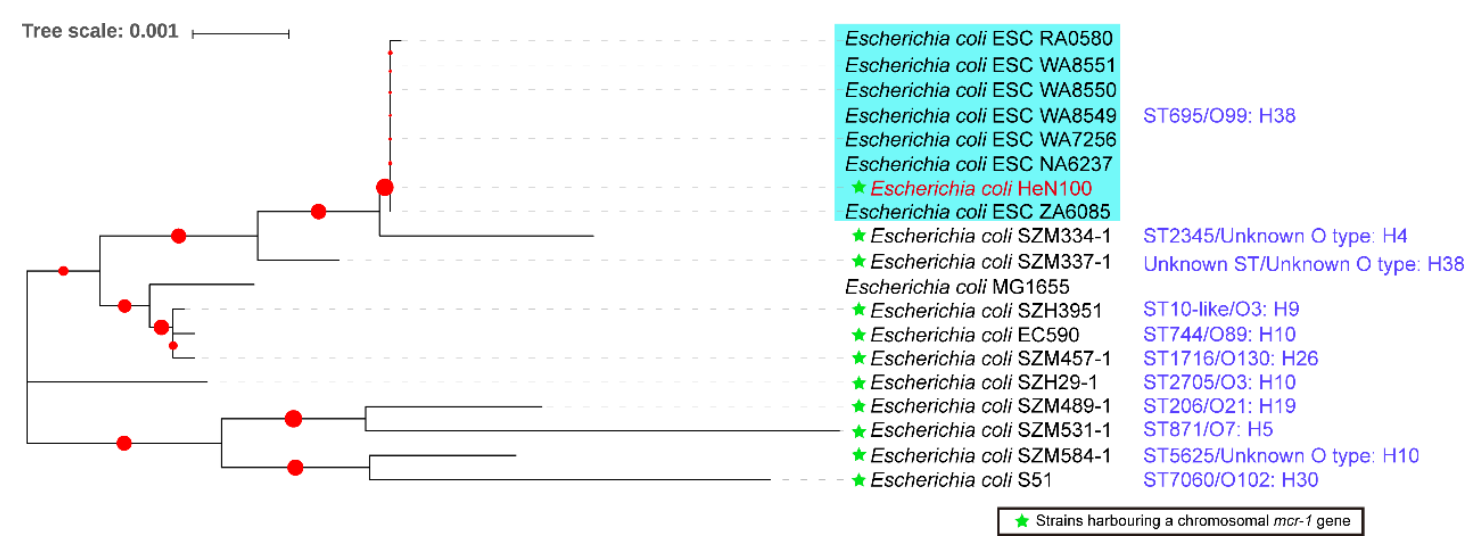

Figure 1. Phylogenetic analysis of E. coli ST695 strains and E. coli strains harboring a chromosomal mor-1 gene. The tree with the highest log likelihood (-14462.49) is shown. The evolutionary history was inferred by using the Maximum Likelihood method and Tamura-Nei model. The percentage of trees in which the associated taxa clustered together is shown next to the branches. Initial tree(s) for the heuristic search were obtained automatically by applying Neighbor-Join and BioNJ algorithms to a matrix of pairwise distances estimated using the Maximum Composite Likelihood (MCL) approach, and then selecting the topology with superior log likelihood value. The tree is drawn to scale, with branch lengths measured in the number of substitutions per site. This analysis involved 19 nucleotide sequences. There were a total of 9015 positions in the final dataset. Evolutionary analyses were conducted in MEGA X. The circles denote bootstrap values within range of 0.015-0.838. 


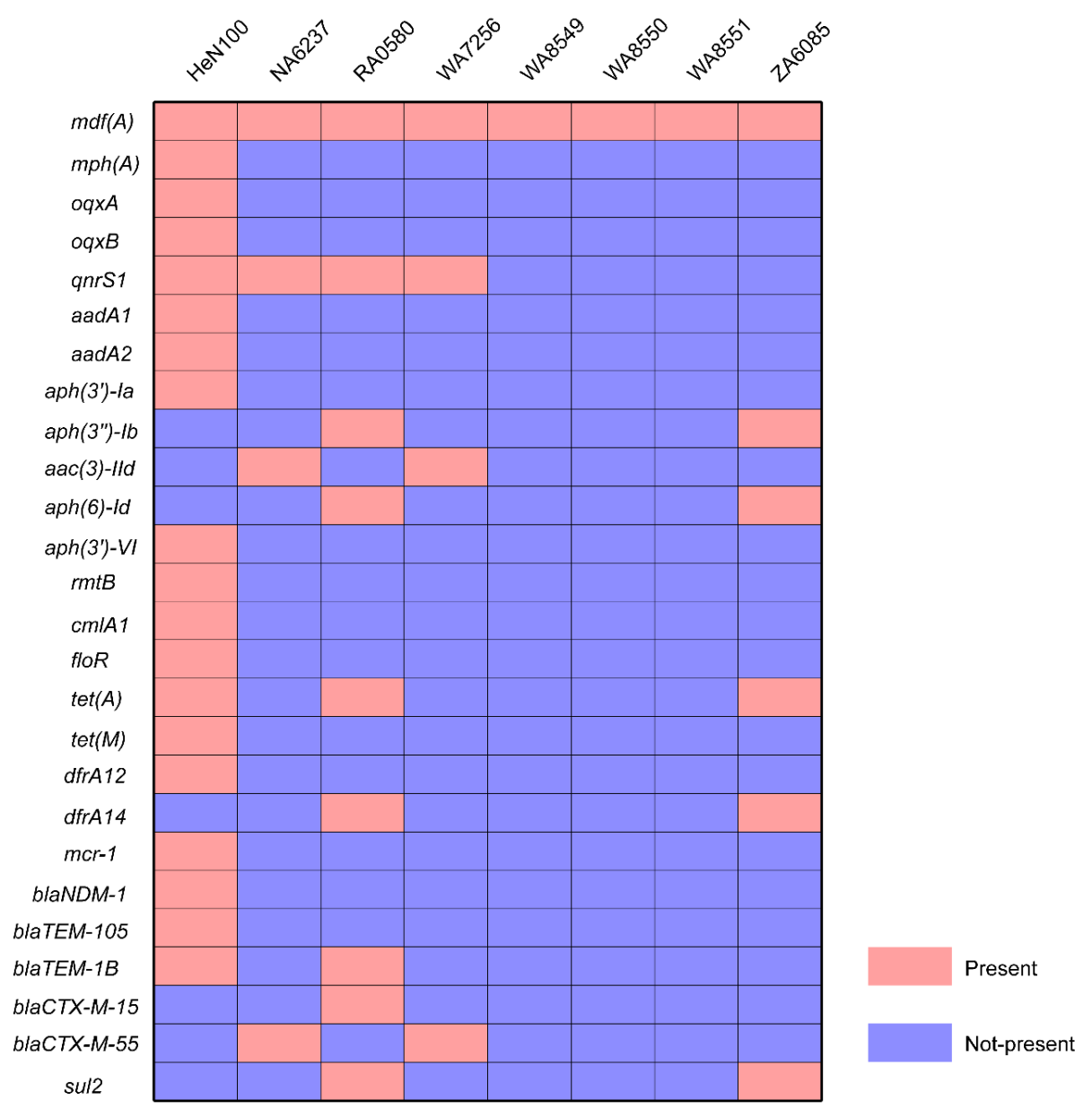

Figure 2. Heatmap showing the presence of antibiotic resistance genes in E. coli ST695. Boxes in pink represent a resistance gene is present in the genome analyzed while boxes in light blue represent a resistance gene is not present in the genome analyzed.

A.

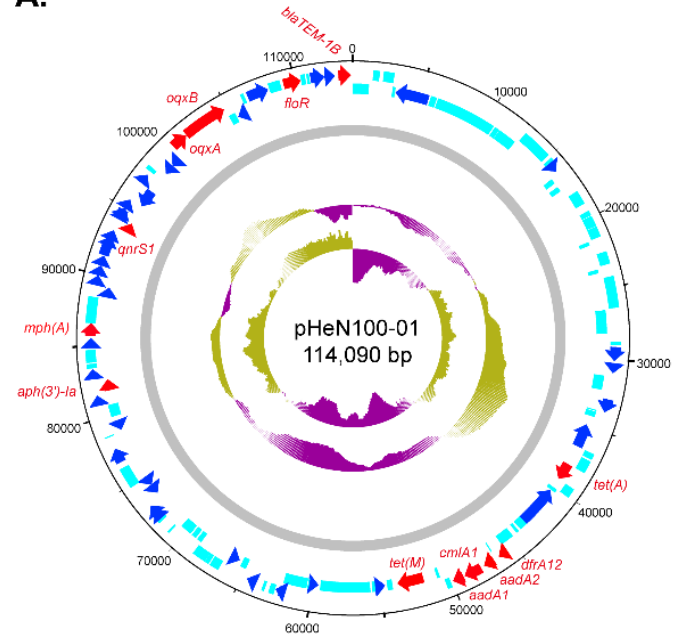

B.

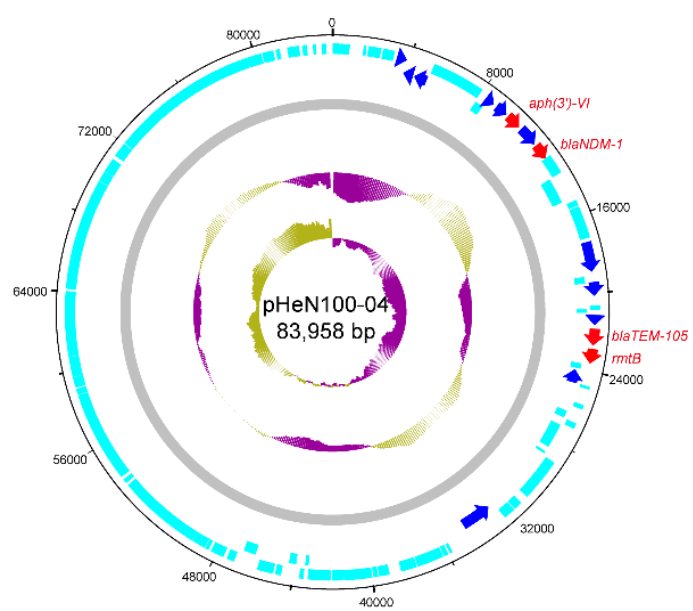

Figure 3. Circle maps of the resistance genes carrying plasmids harbored by the multidrug resistance E. coli HeN100. (A) Circle maps of the resistance plasmid pHeN100-01 carried by HeN100; (B) circle maps of the resistance plasmid pHeN100-04 carried by HeN100. The maps were generated by dnaplotter. Circles from inside to outside showing GC skew (circle 1), GC plot (circle 2), DNA sequence (circle 3), functional genes (circle 4). Resistance genes are displayed as arrows in red while arrows in blue represent putative insertion elements. 


\subsection{Genetic Environment of the Chromosomal mor-1}

The MDR HeN100 contained a $m c r-1$ gene and in particular, this $m c r-1$ was located on the chromosomal genome (Figure 4A). To understand the genetic environment of the chromosomal mcr-1, the $m c r-1$-locating sequences in different E. coli strains were extracted and compared. The results revealed that the $m c r-1$ gene in each of the E. coli strains was located between an IS30 family element ISApl1 (arrow in orange; Figure 4B) and a PAP2 family protein encoding gene (arrows in green; Figure 4B); these three open reading frames (ORFs) consisted of an "ISApl1-mcr-1-orf" structure (Figure 4B). In particularly, one strain, EC590, had three copies of "ISApl1-mcr-1-orf" in its chromosomal genome (Figure 4B). Bioinformatic analysis also revealed that the $m c$-1-bearing segments in HeN100 and the other E. coli strains were inserted in the high AT-rich intergenic regions (Figure 4C).

A.

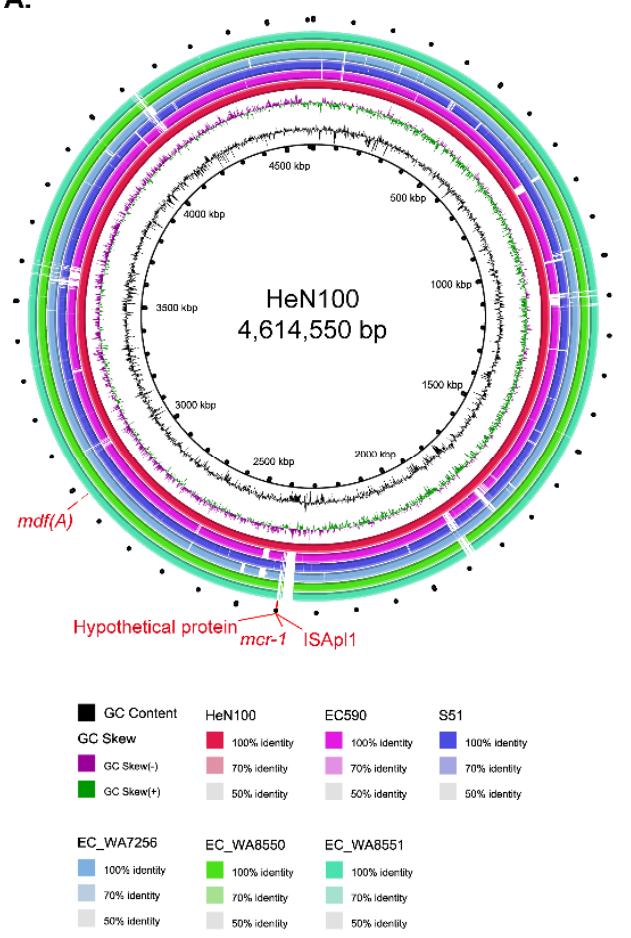

B.

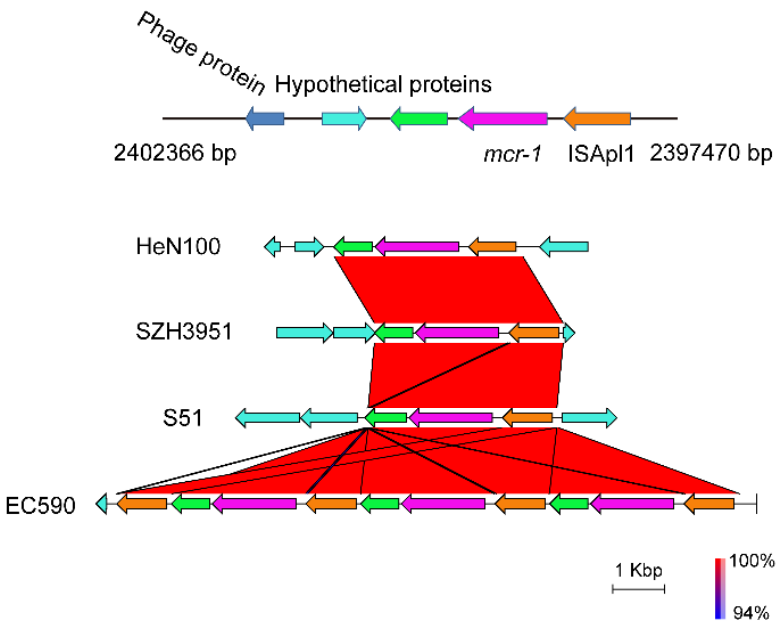

C.

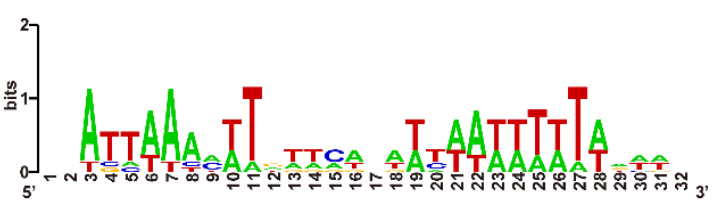

Figure 4. Genetic structure of the chromosomal mcr-1. (A) Genome comparisons of seven E. coli chromosomes. The chromosomal genome sequences of three E. coli strains (HeN100, SZH3951, EC590, S51) harboring the chromosomal $m c r-1$ and three ST695 strains that do not carry mcr-1. Sequence comparison was performed using BRIG package. DNA identities between different sequences are shown in different colors. (B) Genetic structures of chromosomal mor-1 and their comparative analysis among different E. coli strains. Sequence comparison was performed using EasyFig package (version 2.2.2). Color code stands for BLASTn identity of those regions between strains. Arrows in orange, purple, and green represent ISApl1, $m c r-1$, and the linked open reading frame, respectively. (C) The insertion sites of the $m c r-1$ segments in E. coli chromosomes. DNA sequences of $32 \mathrm{bp}$ around the insertion sites (16 bp from both parts) in the four complete genomes were compared and analyzed using WebLogo.

\subsection{Genetic Structure of the Blandm-1-Carrying Plasmid}

Whole genome sequencing also revealed that HeN100 harbored $b l a_{\mathrm{NDM}}-1$-carrying plasmid which was designated pHeN100-04 (Figure 3B). This plasmid was found to be an IncFII type plasmid which was $83,958 \mathrm{bp}$ in size and contained 109 open reading frames. Bioinformatical analysis showed that this plasmid was highly homologous to a E. coli strain plasmid pHNEC55 (GenBank accession no. KT879914; Figure 5) [28]. The average nucleotide identity (AIN) between the two plasmid sequences was $99.96 \%$. The backbone of pHeN100-04 displayed $>99 \%$ homology to that of pHNEC55. However, the multidrug 
resistant (MDR) elements between the two plasmids shared low level of homology (Figure 5). The MDR region of pHNEC55 comprised four resistance genes $b l a_{\mathrm{NDM}-1}, f o s A 3, b l a_{\mathrm{TEM}}$, and $r m t B$, which were flanked by four IS26 elements (Figure 5). The MDR region of pHeN100-04 comprised two antimicrobial resistance gene cassettes, including a 9.9-kb cassette harboring the $a p h\left(3^{\prime}\right)-V I$ and $b l a_{\mathrm{NDM}-1}$ genes and a 5.3-kb cassette harboring the $b l a_{\mathrm{TEM}}$ and $r m t B$ genes (Figure 5). The 9.9-kb cassette was flanked by ISAba14 and ISVsa3, while the 5.3-kb cassette was flanked by two IS26 elements (Figure 5).

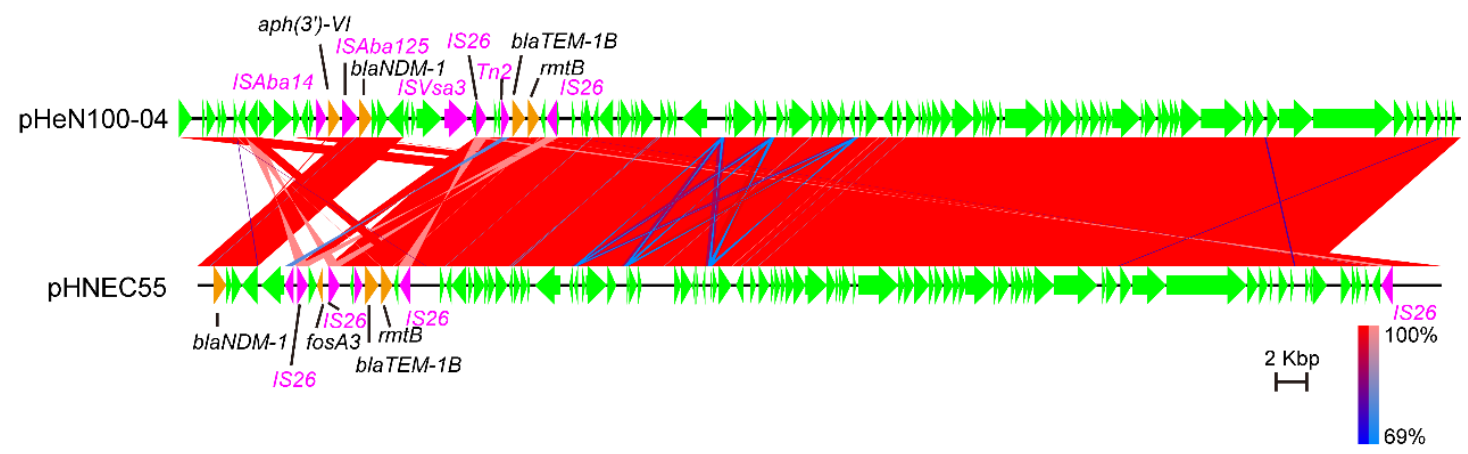

Figure 5. Comparative analysis of plasmids pHeN100-04 and pHNEC55. Sequence comparison was performed using EasyFig package (version 2.2.2). Color code stands for BLASTn identity of those regions between strains. Arrows represent open reading frames encoded by each of the plasmid sequences. Antibiotic resistance genes in each of the plasmid sequences were shown in orange arrows while insertion sequence (IS) elements were shown in purple arrows.

\subsection{The Transferability of the Bla $a_{n d m-1}$-Carrying Plasmid}

To access the transferability of the $b l a_{\mathrm{NDM}-1}$-carrying plasmid pHeN100-04, conjugation experiment was performed between the donor strain $\mathrm{HeN1} 100$ and the recipient strain C600. Conjugation frequency of pHeN100-04 measured using plates supplemented with imipenem was $7.61 \pm 2.11 \times 10^{-5}$ per recipient. Antimicrobial susceptibility testing showed that the transconjugants selected by the selective agars were resistant to the three carbapenems and the other nine $\beta$-lactams, as well as the aminoglycosides tested, suggesting that the conjugation of the plasmid confers resistance to these antibiotics (Table 2).

Table 2. Phenotypical characteristics of the transconjugants selected by rifampin plus imipenem.

\begin{tabular}{ccc}
\hline \multirow{2}{*}{ Antibiotics Tested } & \multicolumn{2}{c}{ Minimum Inhibitory Concentration $(\mu \mathrm{g} / \mathrm{mL})$} \\
\cline { 2 - 3 } & Transconjugants & E. coli C600 \\
\hline Amikacin & $>32(\mathrm{R})$ & $\leq 8(\mathrm{~S})$ \\
Gentamicin & $>8(\mathrm{R})$ & $\leq 2(\mathrm{~S})$ \\
Tobramycin & $>8(\mathrm{R})$ & $\leq 2(\mathrm{~S})$ \\
Ertapenem & $>2(\mathrm{R})$ & $\leq 0.25(\mathrm{~S})$ \\
Imipenem & $>8(\mathrm{R})$ & $0.5(\mathrm{~S})$ \\
Meropenem & $>8(\mathrm{R})$ & $\leq 0.13(\mathrm{~S})$ \\
Cefazolin & $>16(\mathrm{R})$ & $4(\mathrm{~S})$ \\
Cefuroxime & $>16(\mathrm{R})$ & $16(\mathrm{R})$ \\
Cefoxitin & $>16(\mathrm{R})$ & $8(\mathrm{~S})$ \\
Ceftazidime & $>32(\mathrm{R})$ & $\leq 1(\mathrm{~S})$ \\
Ceftriaxone & $>32(\mathrm{R})$ & $\leq 1(\mathrm{~S})$ \\
Cefepime & $16(\mathrm{R})$ & $\leq 1(\mathrm{~S})$ \\
Amoxicillin/clavulanate & $>32 / 16(\mathrm{R})$ & $\leq 8 / 4(\mathrm{~S})$ \\
Ampicillin/sulbactam & $>16 / 8(\mathrm{R})$ & $8 / 4(\mathrm{~S})$ \\
Piperacillin/tazobactam & $>64 / 4(\mathrm{R})$ & $\leq 4 / 4(\mathrm{~S})$ \\
Colistin & $\leq 1(\mathrm{~S})$ & $\leq 1(\mathrm{~S})$ \\
Chloramphenicol & $\leq 4(\mathrm{~S})$ & $\leq 4(\mathrm{~S})$ \\
\hline
\end{tabular}


Table 2. Cont.

\begin{tabular}{ccc}
\hline \multirow{2}{*}{ Antibiotics Tested } & \multicolumn{2}{c}{ Minimum Inhibitory Concentration $(\boldsymbol{\mu g} / \mathbf{m L})$} \\
\cline { 2 - 3 } & Transconjugants & E. coli C600 \\
\hline Moxifloxacin & $\leq 0.5(\mathrm{~S})$ & $\leq 0.5(\mathrm{~S})$ \\
Ciprofloxacin & $\leq 0.5(\mathrm{~S})$ & $\leq 0.5(\mathrm{~S})$ \\
Levofloxacin & $\leq 1(\mathrm{~S})$ & $\leq 1(\mathrm{~S})$ \\
Norfloxacin & $\leq 2(\mathrm{~S})$ & $\leq 2(\mathrm{~S})$ \\
Tetracycline & $\leq 2(\mathrm{~S})$ & $\leq 2(\mathrm{~S})$ \\
Minocycline & $\leq 1(\mathrm{~S})$ & $\leq 1(\mathrm{~S})$ \\
Trimethoprim/sulfamethoxazole & $\leq 1 / 19(\mathrm{~S})$ & $\leq 1 / 19(\mathrm{~S})$ \\
Aztreonam & $\leq 2(\mathrm{~S})$ & $\leq 2(\mathrm{~S})$ \\
Fosfomycin & $\leq 16(\mathrm{~S})$ & $\leq 16(\mathrm{~S})$ \\
Nitrofurantoin & $\leq 16(\mathrm{~S})$ & $\leq 16(\mathrm{~S})$ \\
Tigecycline & $\leq 1(\mathrm{~S})$ & $\leq 1(\mathrm{~S})$ \\
\hline
\end{tabular}

R: Resistant; S: susceptible; I: intermediately resistant.

\section{Discussion}

Several members of polymyxins, particularly colistin, are recognized as the last resort for treating MDR Enterobacteriaceae [29]. The emergence of plasmid-mediated colistin resistance gene mcr-1 raised great concern that the world was on the cusp of the post-antibiotic era [5]. Therefore, almost all epidemiological studies currently published focus on the prevalence and dissemination of plasmid-mediated $m c r-1$ among different species of Enterobacteriaceae [30-34]. Instead, studies on chromosome-born mcr-1 in Enterobacteriaceae are rare. In this study, we recovered an E. coli from the feces of a diarrheal pig and it was determined as ST695. Following E. coli strain RXD100 (GenBank accession no. SQRC00000000) [35], this is the second strain of such sequence type we recovered from diarrheal pigs. While to the best of our knowledge, E. coli ST695 has never been reported in pigs as well as in other animal species, an article has documented one E. coli ST695 from humans (Teresa M. Coque, personal communication) in France expressing extended-spectrum $\beta$-lactamase (ESBLs) CTX-M-15, which displays a broad spectrum of resistance and represents great concern of public health [36]. The ST695 strain HeN100 we recovered from diarrheal pigs presented in this study also showed a broad spectrum of antibiotic resistance (Table 1). In particularly, it was resistant to colistin and twelve $\beta$-lactams including three carbapenems (imipenem, meropenem, ertapenem) tested. The resistant phenotypes of HeN100 was similar to those of RXD100, another ST695 we also isolated from diarrheal pigs [35]. It should be noted that both carbapenems and colistin are the last-resort antibiotics used for treating multidrug-resistant Gram-negative pathogens [29]. Although the epidemiological data for E. coli ST695 are still lacking, the spread of such clone represents a great concern of public health.

It has been documented that $E$. coli is intrinsically susceptible to almost all clinically relevant antimicrobial agents, but this bacterial species has a great capacity to accumulate resistance genes, mostly through horizontal gene transfer [37]. A number of resistance genes were found in HeN100, including oq $x A$, oq $x B$, and qnrS1 which confer resistance to quinolones; aadA1, aadA2, aph(3')-Ia, $a p h\left(3^{\prime}\right)-V I$, and $r m t B$ which confer pan-resistance to aminoglycosides; bla $a_{\mathrm{TEM}-105}$ and $b l a_{\mathrm{TEM}-1 \mathrm{~B}}$ which confer resistance to cephalosporins (belonging to $\beta$-lactams); $b l a_{\mathrm{NDM}-1}$ which confers resistance to carbapenems (belonging to $\beta$-lactams), and $m c r-1$ which confers resistance to colistin (Figure 2). The presence of those acquired genes should be responsible for the resistant phenotypes of HeN100. All of these resistance mechanisms are proposed as the most problematic mechanisms in E. coli and such $E$. coli strains represent a major reservoir of resistance genes that may be responsible for treatment failures in both human and veterinary medicine [37]. It is worthy of note that the pig-sourced E. coli ST695 did not contain the ESBL CTX-M-15 encoding gene, which is carried by the human-sourced ST695 [36]. However, the pig-sourced E. coli ST695 possessed some other ESBLs encoding genes such as the $b l a_{\mathrm{TEM}}$ genes (Figure 2), which have also received some attention $[38,39]$. 
Plasmids as well as other mobile genetic elements (MGEs), such as transposons and gene cassettes in class 1 and 2 integrons, are likely to play a main role in the dissemination of resistance genes in E. coli [37]. In this study, we identified two resistance plasmids in HeN100 (Figure 3). Most of the acquired genes, including those confer resistance to tetracyclines $(\operatorname{tet}(A)$, tet $(M))$, phenicols $(\mathrm{cmlA1}$, floR), trimethoprim

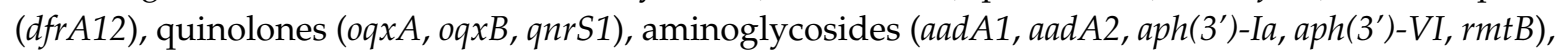
and $\beta$-lactams (bla $\left.a_{\mathrm{NDM}-1}, b l a_{\mathrm{TEM}-105}, b l a_{\mathrm{TEM}-1 \mathrm{~B}}\right)$ (Figure 3$)$, suggesting that the acquisition of those resistance genes in HeN100 is mediated by plasmids. However, the phosphoethanolamine transferase encoding gene in HeN100, the $m c r-1$, which confers resistance to colistin and receives worldwide concern due to its impact on public health, was not carried by a plasmid but was carried by the chromosome (Figures 3 and 4A). This is not consistent with the findings in most of the studies, as mor-1 is generally harbored by plasmids, particularly the IncX4-, IncI2-, IncHI2-, IncP-, IncFII-, and IncY-type plasmids [9]. Bioinformatical analysis revealed the chromosomal $m c r-1$ in HeN100 was adjacent to ISApl1 and a PAP2 family protein encoding gene, and they consisted of a "ISApl1-mcr-1-orf" structure (Figure 4A and 4B). The same structure was also conservatively presented in the other E. coli strains which also have a chromosomally-encoded mcr-1 (Figure 4B). It has been reported that ISApl1 has an intimate relationship with $m c r-1$, and a currently available study on the genetic basis of chromosomally-encoded mcr-1 gene has found that "ISApl1-mor-1-orf" structure is presented in most chromosome-born mcr-1 harbored E. coli [10]. These findings might suggest that insertion of ISApl1 in bacterial chromosomes could be a prerequisite for the mobilization of $m c r-1$-carrying mobile elements on bacterial chromosomes [10]. Indeed, a previous study has experimentally confirmed that $m c r-1$ is always mobilized by two copies of ISApl1 ("ISApl1-mcr-1- ISApl1") [40]. However, most chromosome-born $m c r-1$ only a single copy of ISApl1 at its $5^{\prime}$ extremity (Figure 4B). This might be explained by the characteristic of IS30 family members to excise one copy of the IS element after transposition, in order to stabilize the genetic structure once integrated [40-42]. In addition, most chromosomal $m c r-1$ was found to be inserted in the AT-rich regions (Figure 4C). This is because ISApl1 like other IS30-like elements targets preferentially AT-rich sequences $[40,43]$.

In addition to $m c r-1$, the worldwide dissemination of $b l a_{\mathrm{NDM}-1}$ also represents a great concern of public health [44]. In this study, we found that HeN100 harbored a bla $a_{\text {NDM-1 }}$ which confers the host bacterium resistance to the carbapenems tested, including imipenem, meropenem, and ertapenem (Table 1). This gene was found to be carried by an IncFII type plasmid pHeN100-04 which was highly homologous to pHNEC55 (Figure 5), also an IncFII type plasmid mediating the transmission of $b l a_{\text {NDM-1 }}$ elements among animal-borne E. coli [28]. A previous study has shown that pHNEC55 is conjugative and the dissemination of $b l a_{\mathrm{NDM}-1}$ in $E$. coli from animals was due to the capture of $b l a_{\mathrm{NDM}-1}$-encoding mobile elements by conjugative plasmids that circulate among animal E. coli isolates [28]. Our results also revealed that pHeN100-04 was conjugative and its conjugation frequency was 7.61 $\pm 2.11 \times 10^{-5}$ per recipient. The conjugation of this plasmid conferred the receipt bacteria resistance to carbapenems and other $\beta$-lactams, as well as aminoglycosides (Table 2). The dissemination of such plasmids should receive more attention, as they may be easily disseminated within an animal farm and further transmitted to humans [28].

\section{Conclusions}

In summary, our study presented here reported the phenotypic and genetic characteristics of a chromosome-born mcr-1 carrying E. coli ST695. This isolate displayed a broad spectrum of antibiotic resistance, including resistance to carbapenems and colistin, the last-resort antibiotics used for treating multidrug-resistant Gram-negative pathogens. This strain harbored many acquired genes conferring resistance to these antibiotics, including the two global public health concerned $m c r-1$ and $b l a_{\mathrm{NDM}-1}$, which were located on the chromosomal genome and an IncFII type plasmid, respectively. A "ISApl1-mcr-1-orf" segment inserted in the high AT-rich intergenic regions consisted of the genetic environment of the chromosome-born $m c r-1$. In addition, the $b l a_{\mathrm{NDM}-1}$ was conjugative and its conjugation conferred resistance to carbapenems and some other antibiotics. While pan-epidemiological 
data on the $m c r-1 / b l a_{\mathrm{NDM}-1}$-carrying E. coli ST695 is still lacking, the spread of such E. coli represents a great concern of public health.

Author Contributions: Conceptualization, Z.P., H.C. and X.W.; methodology, Z.P., Z.H., Z.L., X.L., C.J., X.Z.; writing—original draft preparation, Z.P.; writing—review and editing, Z.P., B.W., X.W.; supervision, H.C. and X.W.; project administration, H.C. and X.W.; funding acquisition, H.C. and X.W.

Funding: This research was funded by the National Key Research \& Development Program of China (grant numbers 2017YFC1600103 and 2017YFC1600101); and the earmarked fund for China Agriculture Research System (grant number: CARS-35). Zhong Peng was supported in part by China Postdoctoral Science Foundation (grant number: 2018M640719). The APC was funded by the National Key Research \& Development Program of China.

Acknowledgments: We sincerely acknowledge Teresa M. Coque at Ramón y Cajal Health Research Institute (IRYCIS), Madrid, Spain, for providing the origination information of the human ST695 documented in his publication on Emerg Infect Dis (Reference 36).

Conflicts of Interest: The authors declare no conflict of interest.

\section{References}

1. Trimble, M.J.; Mlynarcik, P.; Kolar, M.; Hancock, R.E. Polymyxin: Alternative Mechanisms of Action and Resistance. Cold Spring Harb. Perspect. Med. 2016, 6. [CrossRef]

2. Poirel, L.; Jayol, A.; Nordmann, P. Polymyxins: Antibacterial Activity, Susceptibility Testing, and Resistance Mechanisms Encoded by Plasmids or Chromosomes. Clin. Microbiol. Rev. 2017, 30, 557-596. [CrossRef]

3. Falagas, M.E.; Rafailidis, P.I.; Matthaiou, D.K. Resistance to polymyxins: Mechanisms, frequency and treatment options. Drug Resist. Updates 2010, 13, 132-138. [CrossRef]

4. Paterson, D.L.; Bonomo, R.A. Multidrug-Resistant Gram-Negative Pathogens: The Urgent Need for 'Old' Polymyxins. Adv. Exp. Med. Biol. 2019, 1145, 9-13. [CrossRef]

5. Yao, X.; Doi, Y.; Zeng, L.; Lv, L.; Liu, J.H. Carbapenem-resistant and colistin-resistant Escherichia coli co-producing NDM-9 and MCR-1. Lancet Infect. Dis. 2016, 16, 288-289. [CrossRef]

6. Li, Z.; Cao, Y.; Yi, L.; Liu, J.H.; Yang, Q. Emergent Polymyxin Resistance: End of an Era? Open Forum. Infect. Dis. 2019, 6. [CrossRef]

7. Moffatt, J.H.; Harper, M.; Boyce, J.D. Mechanisms of Polymyxin Resistance. Adv. Exp. Med. Biol. 2019, 1145, 55-71. [CrossRef]

8. Liu, Y.Y.; Wang, Y.; Walsh, T.R.; Yi, L.X.; Zhang, R.; Spencer, J.; Doi, Y.; Tian, G.; Dong, B.; Huang, X.; et al. Emergence of plasmid-mediated colistin resistance mechanism MCR-1 in animals and human beings in China: A microbiological and molecular biological study. Lancet Infect. Dis. 2016, 16, 161-168. [CrossRef]

9. Doumith, M.; Godbole, G.; Ashton, P.; Larkin, L.; Dallman, T.; Day, M.; Day, M.; Muller-Pebody, B.; Ellington, M.J.; de Pinna, E.; et al. Detection of the plasmid-mediated mcr-1 gene conferring colistin resistance in human and food isolates of Salmonella enterica and Escherichia coli in England and Wales. J. Antimicrob. Chemother. 2016, 71, 2300-2305. [CrossRef]

10. Li, R.; Yu, H.; Xie, M.; Chen, K.; Dong, N.; Lin, D.; Chan, E.W.; Chen, S. Genetic basis of chromosomally-encoded mcr-1 gene. Int. J. Antimicrob. Agents 2018, 51, 578-585. [CrossRef]

11. Wirth, T.; Falush, D.; Lan, R.; Colles, F.; Mensa, P.; Wieler, L.H.; Karch, H.; Reeves, P.R.; Maiden, M.C.; Ochman, H.; et al. Sex and virulence in Escherichia coli: An evolutionary perspective. Mol. Microbiol. 2006, 60, 1136-1151. [CrossRef] [PubMed]

12. Koren, S.; Walenz, B.P.; Berlin, K.; Miller, J.R.; Bergman, N.H.; Phillippy, A.M. Canu: Scalable and accurate long-read assembly via adaptive k-mer weighting and repeat separation. Genome Res. 2017, 27, 722-736. [CrossRef] [PubMed]

13. Walker, B.J.; Abeel, T.; Shea, T.; Priest, M.; Abouelliel, A.; Sakthikumar, S.; Cuomo, C.A.; Zeng, Q.; Wortman, J.; Young, S.K.; et al. Pilon: An integrated tool for comprehensive microbial variant detection and genome assembly improvement. PLoS ONE 2014, 9, e112963. [CrossRef] [PubMed]

14. Tatusova, T.; DiCuccio, M.; Badretdin, A.; Chetvernin, V.; Nawrocki, E.P.; Zaslavsky, L.; Lomsadze, A.; Pruitt, K.D.; Borodovsky, M.; Ostell, J. NCBI prokaryotic genome annotation pipeline. Nucleic Acids Res. 2016, 44, 6614-6624. [CrossRef] [PubMed] 
15. Joensen, K.G.; Tetzschner, A.M.; Iguchi, A.; Aarestrup, F.M.; Scheutz, F. Rapid and Easy in Silico Serotyping of Escherichia coli Isolates by Use of Whole-Genome Sequencing Data. J. Clin. Microbiol. 2015, 53, 2410-2426. [CrossRef]

16. Gibson, M.K.; Forsberg, K.J.; Dantas, G. Improved annotation of antibiotic resistance determinants reveals microbial resistomes cluster by ecology. ISME J. 2015, 9, 207-216. [CrossRef]

17. Zankari, E.; Hasman, H.; Cosentino, S.; Vestergaard, M.; Rasmussen, S.; Lund, O.; Aarestrup, F.M.; Larsen, M.V. Identification of acquired antimicrobial resistance genes. J. Antimicrob. Chemother. 2012, 67, 2640-2644. [CrossRef]

18. Larsen, M.V.; Cosentino, S.; Rasmussen, S.; Friis, C.; Hasman, H.; Marvig, R.L.; Jelsbak, L.; Sicheritz-Ponten, T.; Ussery, D.W.; Aarestrup, F.M.; et al. Multilocus sequence typing of total-genome-sequenced bacteria. J. Clin. Microbiol. 2012, 50, 1355-1361. [CrossRef]

19. Siguier, P.; Perochon, J.; Lestrade, L.; Mahillon, J.; Chandler, M. ISfinder: The reference centre for bacterial insertion sequences. Nucleic Acids Res. 2006, 34, D32-D36. [CrossRef]

20. Rozewicki, J.; Li, S.; Amada, K.M.; Standley, D.M.; Katoh, K. MAFFT-DASH: Integrated protein sequence and structural alignment. Nucleic Acids Res. 2019, 47, W5-W10. [CrossRef]

21. Alikhan, N.F.; Petty, N.K.; Ben Zakour, N.L.; Beatson, S.A. BLAST Ring Image Generator (BRIG): Simple prokaryote genome comparisons. BMC Genom. 2011, 12, 402. [CrossRef] [PubMed]

22. Sullivan, M.J.; Petty, N.K.; Beatson, S.A. Easyfig: A genome comparison visualizer. Bioinformatics 2011, 27, 1009-1010. [CrossRef] [PubMed]

23. Goris, J.; Konstantinidis, K.T.; Klappenbach, J.A.; Coenye, T.; Vandamme, P.; Tiedje, J.M. DNA-DNA hybridization values and their relationship to whole-genome sequence similarities. Int. J. Syst. Evol. Microbiol. 2007, 57, 81-91. [CrossRef] [PubMed]

24. Crooks, G.E.; Hon, G.; Chandonia, J.M.; Brenner, S.E. WebLogo: A sequence logo generator. Genome Res. 2004, 14, 1188-1190. [CrossRef] [PubMed]

25. Kumar, S.; Stecher, G.; Li, M.; Knyaz, C.; Tamura, K. MEGA X: Molecular Evolutionary Genetics Analysis across Computing Platforms. Mol. Biol. Evol. 2018, 35, 1547-1549. [CrossRef]

26. Tamura, K.; Nei, M. Estimation of the number of nucleotide substitutions in the control region of mitochondrial DNA in humans and chimpanzees. Mol. Biol. Evol. 1993, 10, 512-526. [CrossRef]

27. Zhao, Y.; Wang, L.; Zhang, Z.; Feng, J.; Kang, H.; Fang, L.; Jiang, X.; Zhang, D.; Zhan, Z.; Zhou, D.; et al. Structural genomics of pNDM-BTR harboring In191 and Tn6360, and other bla NDM-carrying IncN1 plasmids. Future Microbiol. 2017, 12, 1271-1281. [CrossRef]

28. Lin, D.; Xie, M.; Li, R.; Chen, K.; Chan, E.W.; Chen, S. IncFII Conjugative Plasmid-Mediated Transmission of blaNDM-1 Elements among Animal-Borne Escherichia coli Strains. Antimicrob. Agents Chemother. 2017, 61. [CrossRef]

29. Sun, J.; Yang, R.S.; Zhang, Q.; Feng, Y.; Fang, L.X.; Xia, J.; Li, L.; Lv, X.Y.; Duan, J.H.; Liao, X.P.; et al. Co-transfer of blaNDM-5 and mcr-1 by an IncX3-X4 hybrid plasmid in Escherichia coli. Nat. Microbiol. 2016, 1, 16176. [CrossRef]

30. Hameed, F.; Khan, M.A.; Muhammad, H.; Sarwar, T.; Bilal, H.; Rehman, T.U. Plasmid-mediated mcr-1 gene in Acinetobacter baumannii and Pseudomonas aeruginosa: First report from Pakistan. Rev. Soc. Bras. Med. Trop. 2019, 52, e20190237. [CrossRef]

31. Abdul Momin, M.H.F.; Liakopoulos, A.; Bean, D.C.; Phee, L.M.; Wareham, D.W. A novel plasmid-mediated polymyxin resistance determinant (mcr-1.8) in Escherichia coli recovered from broiler chickens in Brunei Darussalam. J. Antimicrob. Chemother. 2019. [CrossRef] [PubMed]

32. Zajac, M.; Sztromwasser, P.; Bortolaia, V.; Leekitcharoenphon, P.; Cavaco, L.M.; Zietek-Barszcz, A.; Hendriksen, R.S.; Wasyl, D. Occurrence and Characterization of mcr-1-Positive Escherichia coli Isolated from Food-Producing Animals in Poland, 2011-2016. Front. Microbiol. 2019, 10, 1753. [CrossRef] [PubMed]

33. Lei, T.; Zhang, J.; Jiang, F.; He, M.; Zeng, H.; Chen, M.; Wu, S.; Wang, J.; Ding, Y.; Wu, Q. First detection of the plasmid-mediated colistin resistance gene mcr-1 in virulent Vibrio parahaemolyticus. Int. J. Food Microbiol. 2019, 308, 108290. [CrossRef] [PubMed]

34. Martino, F.; Tijet, N.; Melano, R.; Petroni, A.; Heinz, E.; De Belder, D.; Faccone, D.; Rapoport, M.; Biondi, E.; Rodrigo, V.; et al. Isolation of five Enterobacteriaceae species harbouring blaNDM-1 and mcr-1 plasmids from a single paediatric patient. PLoS ONE 2019, 14, e0221960. [CrossRef] 
35. Peng, Z.; Li, X.; Hu, Z.; Li, Z.; Lv, Y.; Lei, M.; Wu, B.; Chen, H.; Wang, X. Characteristics of Carbapenem-Resistant and Colistin-Resistant Escherichia coli Co-Producing NDM-1 and MCR-1 from Pig Farms in China. Microorganisms 2019, 7, 482. [CrossRef]

36. Coque, T.M.; Novais, A.; Carattoli, A.; Poirel, L.; Pitout, J.; Peixe, L.; Baquero, F.; Canton, R.; Nordmann, P. Dissemination of clonally related Escherichia coli strains expressing extended-spectrum beta-lactamase CTX-M-15. Emerg. Infect. Dis. 2008, 14, 195-200. [CrossRef]

37. Poirel, L.; Madec, J.Y.; Lupo, A.; Schink, A.K.; Kieffer, N.; Nordmann, P.; Schwarz, S. Antimicrobial Resistance in Escherichia coli. Microbiol. Spectr. 2018, 6. [CrossRef]

38. Hansen, K.H.; Andreasen, M.R.; Pedersen, M.S.; Westh, H.; Jelsbak, L.; Schonning, K. Resistance to piperacillin/tazobactam in Escherichia coli resulting from extensive IS26-associated gene amplification of blaTEM-1. J. Antimicrob. Chemother. 2019. [CrossRef]

39. Aeksiri, N.; Toanan, W.; Sawikan, S.; Suwannarit, R.; Pungpomin, P.; Khieokhajonkhet, A.; Niumsup, P.R. First Detection and Genomic Insight into mcr-1 Encoding Plasmid-Mediated Colistin-Resistance Gene in Escherichia coli ST101 Isolated from the Migratory Bird Species Hirundo rustica in Thailand. Microb. Drug Resist. 2019. [CrossRef]

40. Poirel, L.; Kieffer, N.; Nordmann, P. In Vitro Study of ISApl1-Mediated Mobilization of the Colistin Resistance Gene mcr-1. Antimicrob. Agents Chemother. 2017, 61. [CrossRef]

41. Szabo, M.; Kiss, J.; Kotany, G.; Olasz, F. Importance of illegitimate recombination and transposition in IS30-associated excision events. Plasmid 1999, 42, 192-209. [CrossRef] [PubMed]

42. Szabo, M.; Kiss, J.; Nagy, Z.; Chandler, M.; Olasz, F. Sub-terminal sequences modulating IS30 transposition in vivo and in vitro. J. Mol. Biol. 2008, 375, 337-352. [CrossRef] [PubMed]

43. Tegetmeyer, H.E.; Fricke, K.; Baltes, N. An isogenic Actinobacillus pleuropneumoniae AasP mutant exhibits altered biofilm formation but retains virulence. Vet. Microbiol. 2009, 137, 392-396. [CrossRef] [PubMed]

44. Wu, W.; Feng, Y.; Tang, G.; Qiao, F.; McNally, A.; Zong, Z. NDM Metallo-beta-Lactamases and Their Bacterial Producers in Health Care Settings. Clin. Microbiol. Rev. 2019, 32. [CrossRef] [PubMed]

(C) 2019 by the authors. Licensee MDPI, Basel, Switzerland. This article is an open access article distributed under the terms and conditions of the Creative Commons Attribution (CC BY) license (http://creativecommons.org/licenses/by/4.0/). 